\title{
Effects of safflower seed extract on arterial stiffness
}

This article was published in the following Dove Press journal:

Vascular Health and Risk Management

2 November 2010

Number of times this article has been viewed

\section{Katsuya Suzuki' \\ Shigekazu Tsubaki ${ }^{2}$ \\ Masami Fujita ${ }^{3}$ \\ Naoto Koyama' \\ Michio Takahashi' \\ Kenji Takazawa ${ }^{4}$}

'Research Institute for Health Fundamentals, Ajinomoto Co., Inc., Kawasaki; ${ }^{2}$ Samoncho Clinic, Tokyo; ${ }^{3}$ Shinanozaka Clinic, Tokyo; ${ }^{4}$ Tokyo Medical University Hachioji Medical Center, Tokyo, Japan
Correspondence: Katsuya Suzuki Research Institute for Health Fundamentals, Ajinomoto Co., Inc. I - I, Suzuki-cho, Kawasaki-ku, Kawasaki

210-868I, Japan

Tel +8I 442105767

Fax $+8 \mid 442117609$

Email katsuya_suzuki@ajinomoto.com
Abstract: Safflower seed extract (SSE) contains characteristic polyphenols and serotonin derivatives ( $\mathrm{N}-(p$-coumaroyl) serotonin and $\mathrm{N}$-feruloylserotonin), which are reported to inhibit oxidation of low-density lipoprotein (LDL), formation of atherosclerotic plaques, and improve arterial stiffness as assessed by pulse wave analysis in animal models. The effects of long-term supplementation with SSE on arterial stiffness in human subjects were evaluated. This doubleblind, placebo-controlled study was conducted in 77 males (35-65 years) and 15 postmenopausal females (55-65 years) with high-normal blood pressure or mild hypertension who were not undergoing treatment. Subjects received SSE (70 mg/day as serotonin derivatives) or placebo for 12 weeks, and pulse wave measurements, ie, second derivative of photoplethysmogram (SDPTG), augmentation index, and brachial-ankle pulse wave velocity (baPWV) were conducted at baseline, and at weeks 4,8 , and 12 . Vascular age estimated by SDPTG aging index improved in the SSE-supplemented group when compared with the placebo group at four $(P=0.0368)$ and 12 weeks $(P=0.0927)$. The trend of augmentation index reduction ( $P=0.072$ versus baseline) was observed in the SSE-supplemented group, but reduction of baPWV by SSE supplementation was not observed. The SSE-supplemented group also showed a trend towards a lower malondialdehyde-modified-LDL autoantibody titer at 12 weeks from baseline. These results suggest long-term ingestion of SSE in humans could help to improve arterial stiffness.

Keywords: safflower, serotonin derivatives, antioxidants, augmentation index, pulse wave velocity

\section{Introduction}

In Korea and other Asian countries, safflower (Carthamus tinctorius L.) seeds have been used as a traditional food or herbal medicine for the treatment of rheumatism and osteoporotic fractures. ${ }^{1,2}$ Among the polyphenols, two major serotonin derivatives, $N$-( $p$-coumaroyl) serotonin (CS) and $N$-feruloylserotonin (FS), have been identified as the characteristic components of defatted safflower seed extract (SSE). ${ }^{3-5}$ Serotonin derivatives have been shown to have potent antioxidant property, ${ }^{4,5}$ antitumor activity, ${ }^{1,6}$ fibroblast proliferation stimulating activity, ${ }^{7}$ and protective effects against postischemic myocardial dysfunction. ${ }^{8} \mathrm{SSE}$, as well as serotonin derivatives, have been reported to have the ability to reduce atherosclerotic lesions in apolipoprotein E-deficient mice and in Kurosawa and Kusanagi-hypercholesterolemic (KHC) rabbits. ${ }^{5,9}$ Katsuda et al reported that SSE or serotonin derivatives reduced pulse wave velocity (a clinical parameter of noninvasive arterial stiffness) and ameliorated the pressure-strain elastic modulus (index of distension of the wall) in the aorta of KHC rabbits. ${ }^{9}$ 
Parameters of arterial stiffness are associated with aging and increase the relative risk of atherosclerosis. Noninvasive pulse wave analysis methods, such as pulse wave velocity, augmentation index, and second derivative of the fingertip photoplethysmogram (SDPTG) have been developed to measure arterial stiffness. These methods have been postulated to predict cardiovascular events. ${ }^{10-17}$ Several clinical studies have reported the use of drugs for the treatment of arterial stiffness. However, there are few reports on the use of functional food ingredients for this purpose. In a human pilot study, SSE supplementation was found to have a favorable effect on brachial-ankle pulse wave velocity (baPWV) in individuals with relatively high blood pressure (BP) or with a relatively high baPWV. ${ }^{18}$ Therefore, we investigated the effects of SSE on arterial stiffness in males and postmenopausal females with high-normal BP or mild hypertension. The endpoints were baPWV, augmentation index at the radial artery, and SDPTG.

\section{Material and methods}

This study was conducted according to the guidelines laid down in the Declaration of Helsinki, and all procedures involving human subjects were approved by the Clinical Trial Ethics Committee of the Shinanozaka Clinic, Tokyo, Japan. Written informed consent was obtained from all subjects.

\section{Study protocol and subjects}

This 12-week, double-blind, placebo-controlled study was conducted in 77 males (35-65 years) and 15 postmenopausal females (55-65 years) with high-normal BP or mild hypertension. Before the main clinical examination, two prior measurements were carried out in enrolled subjects according to the following inclusion criteria: baPWV $>1250 \mathrm{~cm} / \mathrm{sec}$ and rate of baPWV change between the two prior measurements being $<7 \%$. Subjects with a history or presence of cardiovascular disease, on medication for hypertension, hyperlipidemia, or diabetes mellitus, or medication for inflammatory disease, such as arthritis, dermatitis, and hepatitis, were excluded from the study. Subjects received three capsules of SSE (11.7 mg of serotonin derivatives [CS + FS] per capsule) or placebo (180 mg of lactose per capsule) twice a day for 12 weeks (total daily serotonin derivative intake $70 \mathrm{mg} /$ day). Measurements of pulse wave analysis at baseline, and at weeks 4, 8 and 12, and blood sampling at weeks 0 and 12 were conducted in the morning after an overnight fast. Measurements were conducted twice and averaged to provide a daily mean value. During the treatment period, subjects were advised not to take medicines, antioxidant supplements, polyunsaturated fatty acids, and food for specific lowering of high BP.

\section{Preparation of SSE}

Safflower seeds were provided by ADRI Limited (Sofia, Bulgaria). They were defatted by compression and extracted with $50 \%(\mathrm{v} / \mathrm{v})$ ethanol. Extracted solutions were filtered and concentrated in vacuo. Water was added to the concentrated solution; this was kept overnight at room temperature. The precipitate was mixed with the eluted supernatant, which was loaded onto the adsorption column. The precipitate was then eluted using 50\% (v/v) ethanol. After concentration in vacuo, the samples were freeze-dried. The obtained solid was crushed and filtered. Macronutrient and serotonin derivative compositions of the SSE are shown in Table 1. The SSE powder was filled in hard gelatin capsules and administered as an experimental dietary supplement.

\section{Measurement procedures}

BP and baPWV were measured simultaneously using an automated device, ie, pulse wave velocity/ABI (BP-203RPE II; Omron Colin, Tokyo, Japan). The detailed methodology for measuring baPWV has been described elsewhere. ${ }^{18}$ In brief, four pneumatic pressure cuffs, two electrocardiographic electrodes, and a microphone for detecting heart sounds were attached to both the arms and ankles, both wrists, and on the left edge of the sternum to record the volume waveform for the brachial and ankle arteries. baPWV was automatically calculated as the length of an arterial segment between the brachium and ankle, which was automatically calculated from body height divided by transit time of the pulse wave. The mean of the left- and right-sided measurements of baPWV and BP were calculated to obtain a daily value. The method was validated in a previous report, and the inter- and intraobserver coefficients of variation were $8.4 \%$ and $10.0 \%$, respectively. ${ }^{19}$

Table I Composition of safflower seed extract

\begin{tabular}{ll}
\hline & SSE \\
\hline Energy $(\mathrm{k} / / 100 \mathrm{~g})$ & 1703.7 \\
Protein $(\mathrm{g} / 100 \mathrm{~g})$ & 19.1 \\
Fat $(\mathrm{g} / \mathrm{l00} \mathrm{g})$ & 6.2 \\
Carbohydrate $(\mathrm{g} / 100 \mathrm{~g})$ & 68.0 \\
Ash $(\mathrm{g} / 100 \mathrm{~g})$ & 3.0 \\
Dietary fiber $(\mathrm{g} / 100 \mathrm{~g})$ & 1.3 \\
$\mathrm{CS}(\mathrm{mg} / \mathrm{g})$ & 34.9 \\
FS $(\mathrm{mg} / \mathrm{g})$ & 49.3 \\
\hline
\end{tabular}

Abbreviations: CS, $\mathrm{N}$-(p-coumaroyl) serotonin; FS, $\mathrm{N}$-feruloylserotonin; SSE, safflower seed extract. 
Radial augmentation index was measured and calculated from the pulse wave using a semiautomatic pulse wave analyzer (HEM-9000 AI; Omron Colin). In brief, the arterial pulse wave was noninvasively obtained from the left radial artery by tonometric tracing. The $8 \mathrm{~mm}$ wide tonometric sensor comprised 40 arrayed microtransducers. The most appropriate microtransducer was automatically selected for optimal measurement. The hold-down pressure of the sensor head was automatically adjusted for each subject. Pulse waves were measured for $30 \mathrm{sec}$ and digitized at $500 \mathrm{~Hz}$. Radial augmentation index was calculated as the ratio of the late systolic pressure to pulse pressure, ie:

$$
\begin{aligned}
& \text { Late systolic BP }- \text { diastolic BP/(systolic BP } \\
& - \text { diastolic BP }) \times 100(\%)
\end{aligned}
$$

Radial augmentation index was automatically calculated using a fourth-order differential equation for a radial arterial waveform (HEM-9000 AI). Measurement was carried out with subjects in the sitting position. The coefficients of variation for intra- and interobserver measurements for the radial aging index using this device were $4.3 \%$ and $2.5 \%$, respectively. ${ }^{20}$

SDPTG was measured by fingertip photoplethysmography (SDP-100; Fukuda Denshi, Tokyo, Japan). The sensor was placed on the cuticle of the second digit of the left hand. During the measurement, subjects rested on a bed in a quiet room. The SDPTG consists of five specific waves, ie, a, b, c, and d systolic waves, and an e wave that is diastolic. Ratios for the following parameters were obtained: $b / a, d / a$, and SDPTG aging index (SDPTG-AI; $(b-c-d-e) / a)$. Vascular age was calculated for males as $43.5 \times$ SDPTG-AI +65.9 years and for females as $41.7 \times$ SDPTG-AI +61.8 years. ${ }^{15}$ Reproducibility of the SDPTG has been previously reported, with an intraobserver repeatability of $8 \%{ }^{21}$

\section{Biochemical parameters}

Plasma glucose and serum lipids, ie, total cholesterol, lowdensity lipoprotein (LDL) cholesterol, high-density lipoprotein (HDL) cholesterol, and triglycerides, were measured at SRL Inc. (Tokyo, Japan) using its standard laboratory protocols.

\section{Oxidative stress status}

Serum autoantibody titers to malondialdehyde-modified LDL (anti-MDA-LDL) were measured by enzyme-linked immunosorbent assay (ELISA). ${ }^{18}$ Oxidized LDLs in serum were measured as 4E6 monoclonal antibody-reactive substances using an oxidized LDL competitive ELISA kit (Mercodia, Uppsala, Sweden). The parameters were measured at SRL Inc.

\section{Proinflammatory markers}

The serum-soluble form of vascular cell adhesion molecule-1 (sVCAM-1), the soluble form of intercellular adhesion molecule-1 (sICAM-1), and monocyte chemotactic protein-1 (MCP-1) were measured at SRL Inc. using Quantikine Immunoassay kits (R\&D Systems, Minneapolis, MN).

\section{Statistical analysis}

Results are represented as mean \pm standard deviation unless otherwise stated. All data analyses were conducted using the Statistical Software for Social Sciences (15.0.1 (SPSS, Inc., Chicago, IL). Changes from baseline between the groups were compared using analysis of covariance, adjusting for each baseline result. Comparison with baseline ( 0 week) in the same group was conducted by paired $t$-test.

\section{Results \\ Subject characteristics}

Clinical characteristics of subjects included in this study are presented in Table 2. Before administration of the test material, baPWV in the placebo group was significantly higher than that in the SSE-supplemented group $(P=0.0231)$. Other factors were not significantly different between the two groups. None of the subjects had serious conditions, such as pulmonary or renal disease.

During the study, three subjects in the SSE-supplemented group were excluded from analysis (two due to failure to

\begin{tabular}{|c|c|c|c|}
\hline & $\begin{array}{l}\text { Placebo group } \\
(n=46)\end{array}$ & $\begin{array}{l}\text { SSE group } \\
(n=46)\end{array}$ & $P$ \\
\hline Male & 39 & 38 & 1.0000 \\
\hline Female & 7 & 8 & \\
\hline Age $(y)$ & $51.6 \pm 7.2$ & $50.6 \pm 8.1$ & 0.5055 \\
\hline Body height $(\mathrm{cm})$ & $168.22 \pm 7.11$ & $168.98 \pm 7.22$ & 0.6108 \\
\hline Body weight (kg) & $67.49 \pm 8.81$ & $69.03 \pm 11.62$ & 0.4760 \\
\hline Body mass index (\%) & $23.81 \pm 2.40$ & $24.12 \pm 3.43$ & 0.6169 \\
\hline baPWV (cm/sec) & $1449.2 \pm 125.0$ & $1390.5 \pm 118.5$ & 0.0231 \\
\hline \multicolumn{4}{|c|}{ Brachial blood pressure $(\mathrm{mmHg})$} \\
\hline SBP & $131.7 \pm 13.3$ & $127.0 \pm 9.8$ & 0.0588 \\
\hline DBP & $83.4 \pm 8.3$ & $81.5 \pm 7.5$ & 0.2478 \\
\hline $\mathrm{b} / \mathrm{a}$ & $-0.545 \pm 0.113$ & $-0.563 \pm 0.155$ & 0.5139 \\
\hline$d / a$ & $-0.362 \pm 0.091$ & $-0.330 \pm 0.104$ & 0.1219 \\
\hline SDPTG-AI & $-0.254 \pm 0.264$ & $-0.318 \pm 0.326$ & 0.3045 \\
\hline Vascular age (y) & $54.5 \pm 11.2$ & $52.1 \pm 13.8$ & 0.3578 \\
\hline Alx (\%) & $83.7 \pm 12.8$ & $81.1 \pm 12.6$ & 0.3446 \\
\hline
\end{tabular}

Table 2 Subject characteristics

Notes: Results are mean \pm standard deviation. Probability value refers to the difference between the treatment groups.

Abbreviations: baPWV, brachial-ankle pulse wave velocity; SBP, systolic blood pressure; DBP, diastolic blood pressure; SDPTG-AI, second derivative of a finger photoplethysmogram aging index; Alx, augmentation index. 
attend the study, and one due to development of hyperuricemia). Statistical analysis of the SSE-supplemented group was conducted in 44 and 43 subjects after eight and 12 weeks of SSE supplementation, respectively.

\section{Effects of SSE on BP and arterial stiffness}

Table 3 shows values and changes in least-squares mean of BP and arterial stiffness parameters from baseline, after ingestion of test meals at weeks 4, 8, and 12. At four weeks, the SSE-supplemented group showed lower SDPTG-AI and $\mathrm{b} / \mathrm{a}(P=0.0620$ and 0.0507 , respectively) than the placebo group. Vascular age was significantly lower in the SSEsupplemented group at four weeks $(P=0.0368)$ and remained significantly lower at 12 weeks $(P=0.0927)$ than in the placebo group. There were no significant differences in systolic BP, diastolic BP, baPWV, and augmentation index between the two groups.

\section{Blood analysis}

Biochemical parameters, oxidative stress, and proinflammatory markers in blood at baseline and 12 weeks after ingestion of test meals are presented in Table 4. Significant variations in LDL cholesterol at 12 weeks from baseline were observed in the SSE-supplemented group. There was no significant change from baseline for other biochemical parameters in either group at 12 weeks. No significant changes in oxidative

Table 3 Effects of SSE supplementation on arterial stiffness and blood pressure

\begin{tabular}{|c|c|c|c|c|c|c|}
\hline & \multirow[t]{2}{*}{ Week } & \multicolumn{2}{|c|}{ Measurement value } & \multicolumn{3}{|c|}{ Least-squares mean change from baseline $(95 \% \mathrm{Cl})$} \\
\hline & & Placebo group & SSE group & Placebo group & SSE group & $\mathbf{P}$ \\
\hline \multicolumn{7}{|c|}{ Brachial blood pressure $(\mathrm{mmHg})$} \\
\hline \multirow[t]{4}{*}{ SBP } & Baseline & $131.7 \pm 13.3$ & $127.0 \pm 9.8$ & & & \\
\hline & 4 & $130.9 \pm 12.1$ & $127.3 \pm 8.7$ & $0.00(-2.12$ to 2.12$)$ & & 0.7391 \\
\hline & 8 & $129.3 \pm 12.0$ & $126.7 \pm 9.2$ & $-1.74(-3.92$ to 0.44$)$ & -1.51 ( $(-3.74$ to 0.72$)$ & 0.8819 \\
\hline & 12 & $129.8 \pm 1 \mid .3$ & $126.2 \pm 9.1$ & $-1.28(-3.27$ to 0.72$)$ & $-2.09(-4.16$ to -0.02$)$ & $0.578 \mathrm{I}$ \\
\hline \multirow[t]{4}{*}{ DBP } & Baseline & $83.4 \pm 8.3$ & $81.5 \pm 7.5$ & & & \\
\hline & 4 & $83.6 \pm 8.8$ & $81.1 \pm 7.1$ & 0.39 (-I.04 to I.82) & $-0.49(-1.92$ to 0.94$)$ & 0.3880 \\
\hline & 8 & $83.0 \pm 9.3$ & $81.1 \pm 7.8$ & $-0.27(-1.88$ to 1.34$)$ & $-0.75(-2.39$ to 0.90$)$ & 0.6827 \\
\hline & 12 & $82.7 \pm 7.8$ & $80.1 \pm 8.0$ & $-0.48(-2.02$ to 1.05$)$ & $-1.75(-3.34$ to -0.17$)$ & 0.2570 \\
\hline \multirow[t]{4}{*}{ baPWV (cm/s) } & Baseline & $1449.2 \pm 125.0$ & $1390.5 \pm 1 \mid 8.5$ & & & \\
\hline & 4 & $|468.2 \pm 15| .8$ & $1420.0 \pm 1 \mid 8.7$ & 23.36 (-2.98 to $49.7 \mathrm{I})$ & 25.21 (-I.I4 to 51.56$)$ & 0.9231 \\
\hline & 8 & $1455.0 \pm 129.4$ & $1408.9 \pm 126.5$ & $10.76(-13.49$ to 35.01$)$ & $9.43(-15.38$ to 34.24$)$ & 0.9402 \\
\hline & 12 & $1440.0 \pm 116.8$ & $1389.6 \pm 110.2$ & $-1.20(-24.08$ to 21.67$)$ & $-14.65(-38.33$ to 9.03$)$ & 0.4246 \\
\hline \multirow[t]{4}{*}{$\mathrm{b} / \mathrm{a}$} & Baseline & $-0.545 \pm 0.113$ & $-0.563 \pm 0.155$ & & & \\
\hline & 4 & $-0.526 \pm 0.114$ & $-0.575 \pm 0.153$ & $0.021(-0.005$ to 0.046$)$ & $-0.016(-0.04 I$ to 0.010$)$ & 0.0507 \\
\hline & 8 & $-0.54 I \pm 0.11 I$ & $-0.571 \pm 0.133$ & $0.006(-0.018$ to 0.030$)$ & $-0.017(-0.04 \mid$ to 0.008$)$ & 0.1877 \\
\hline & 12 & $-0.534 \pm 0.127$ & $-0.572 \pm 0.145$ & $0.011(-0.013$ to 0.036$)$ & $-0.018(-0.044$ to 0.008$)$ & 0.1049 \\
\hline \multirow[t]{4}{*}{$d / a$} & Baseline & $-0.362 \pm 0.091$ & $-0.330 \pm 0.104$ & & & \\
\hline & 4 & $-0.379 \pm 0.092$ & $-0.34 \mathrm{I} \pm 0.103$ & $-0.022(-0.04 I$ to -0.002$)$ & $-0.005(-0.025$ to 0.015$)$ & 0.2413 \\
\hline & 8 & $-0.362 \pm 0.095$ & $-0.334 \pm 0.108$ & $-0.004(-0.027$ to 0.019$)$ & $0.004(-0.019$ to 0.028$)$ & 0.6163 \\
\hline & 12 & $-0.362 \pm 0.108$ & $-0.320 \pm 0.126$ & $-0.003(-0.028$ to 0.023$)$ & $0.018(-0.009$ to 0.045$)$ & 0.2756 \\
\hline \multirow[t]{4}{*}{ SDPTG-AI } & Baseline & $-0.2539 \pm 0.264 I$ & $-0.3178 \pm 0.3262$ & & & \\
\hline & 4 & $-0.1990 \pm 0.2428$ & $-0.3139 \pm 0.3274$ & $0.06 \mathrm{I}(-0.0 \mathrm{II}$ to $0.1 \mathrm{II})$ & $-0.006(-0.057$ to 0.044$)$ & 0.0620 \\
\hline & 8 & $-0.2409 \pm 0.2582$ & $-0.3191 \pm 0.3010$ & $0.020(-0.033$ to 0.073$)$ & $-0.022(-0.077$ to 0.032$)$ & 0.2740 \\
\hline & 12 & $-0.2384 \pm 0.274 \mathrm{I}$ & $-0.3373 \pm 0.3177$ & 0.021 (0.024 to 0.076$)$ & $-0.032(-0.088$ to 0.024$)$ & 0.1880 \\
\hline \multirow[t]{4}{*}{ Vascular age (y) } & Baseline & $54.5 \pm 11.2$ & $52.1 \pm 13.8$ & & & \\
\hline & 4 & $57.1 \pm 10.1$ & $52.0 \pm 13.8$ & 2.83 (0.59 to 5.06$)$ & $-0.57(-2.83$ to 1.69$)$ & 0.0368 \\
\hline & 8 & $55.5 \pm 11.1$ & $51.7 \pm 12.4$ & $1.24(-1.13$ to $3.6 \mathrm{I})$ & $-1.25(-3.67$ to 1.17$)$ & 0.1473 \\
\hline & 12 & $55.4 \pm 11.5$ & $51.3 \pm 13.5$ & $1.05(-1.24$ to 3.34$)$ & $-1.77(-4.14$ to 0.60$)$ & 0.0927 \\
\hline \multirow[t]{4}{*}{ Alx (\%) } & Baseline & $83.7 \pm 12.8$ & $81.1 \pm 12.6$ & & & \\
\hline & 4 & $82.6 \pm 13.6$ & $80.5 \pm 11.9$ & $-0.89(-2.9 \mid$ to 1.14$)$ & $-0.95(-3.00$ to 1.10$)$ & 0.9652 \\
\hline & 8 & $84.1 \pm 13.1$ & $80.4 \pm 11.8$ & $0.68(-1.59$ to 2.95$)$ & $-1.62(-3.94$ to 0.71$)$ & 0.1646 \\
\hline & 12 & $82.5 \pm 12.7$ & $79.6 \pm 12.8$ & $-1.03(-3.08$ to 1.02$)$ & $-2.14(-4.27$ to -0.02$)$ & 0.4561 \\
\hline
\end{tabular}

Notes: Results are mean \pm standard deviation. Probability value refers to the difference between the treatment groups.

Abbreviations: SBP, systolic blood pressure; DBP, diastolic blood pressure; baPWV, brachial-ankle pulse wave velocity; SDPTG-AI, second derivative of a finger photoplethysmogram aging index; Alx, augmentation index; SSE, safflower seed extract; $\mathrm{Cl}$, confidence interval. 
Table 4 Results according to treatment in each group

\begin{tabular}{|c|c|c|c|c|c|}
\hline & & \multicolumn{2}{|l|}{ Placebo } & \multicolumn{2}{|l|}{ SSE } \\
\hline & & $0 w$ & $12 \mathrm{w}$ & $0 \mathrm{w}$ & $12 \mathrm{w}$ \\
\hline Total cholesterol & $(\mathrm{mg} / \mathrm{dL})$ & $205.9 \pm 29.7$ & $202.9 \pm 32.8$ & $214.8 \pm 28.8$ & $209.6 \pm 14.8$ \\
\hline LDL cholesterol & $(\mathrm{mg} / \mathrm{dL})$ & $125.8 \pm 28.3$ & $122.2 \pm 31.6$ & $131.9 \pm 27.0$ & $124.2 \pm 25.3^{*}$ \\
\hline HDL cholesterol & $(\mathrm{mg} / \mathrm{dL})$ & $54.8 \pm 14.3$ & $54.0 \pm 12.5$ & $57.7 \pm 16.5$ & $56.8 \pm 15.3$ \\
\hline Triglycerides & $(\mathrm{mg} / \mathrm{dL})$ & $119.6 \pm 62.2$ & $108.8 \pm 65.6$ & $114.9 \pm 57.7$ & $109.5 \pm 74.1$ \\
\hline Glucose & $(\mathrm{mg} / \mathrm{dL})$ & $102.1 \pm 10.0$ & $102.8 \pm 14.1$ & $100.3 \pm 8.9$ & $101.4 \pm 8.7$ \\
\hline sICAM-I & $(\mathrm{ng} / \mathrm{mL})$ & $207.3 \pm 57.8$ & $201.0 \pm 57.8$ & $184.8 \pm 62.7$ & $177.2 \pm 60.3$ \\
\hline sVCAM-I & $(\mathrm{ng} / \mathrm{mL})$ & $586.7 \pm 153.6$ & $607.8 \pm 162.6$ & $548.0 \pm 178.7$ & $573.9 \pm 137.4$ \\
\hline MCP-I & $(\mathrm{pg} / \mathrm{mL})$ & $255.7 \pm 69.5$ & $251.1 \pm 58.1$ & $270.5 \pm 72.3$ & $256.9 \pm 66.2$ \\
\hline Oxidized LDL & $(\mathrm{U} / \mathrm{L})$ & $73.2 \pm 12.9$ & $72.3 \pm 14.8$ & $74.8 \pm 12.9$ & $71.4 \pm 11.9$ \\
\hline Anti-MDA-LDL & $(\mathrm{AU})$ & $150.52 \pm 63.10$ & $|4| .23 \pm 61.24$ & $175.70 \pm 131.04$ & $138.75 \pm 61.82$ \\
\hline
\end{tabular}

Notes: Results are mean \pm standard deviation. Probability value refers to the comparison of the value at baseline $(0 \mathrm{~W})(* \mathrm{P}<0.05)$, as revealed by paired $t$-test.

Abbreviations: sICAM-I, soluble intercellular adhesion molecule-I; sVCAM-I, soluble vascular cell adhesion molecule-I; MCP-I, monocyte chemotactic protein-I; anti MDA-LDL, autoantibody titers to malondialdehyde-modified LDL; AU, arbitrary unit; SD, standard deviation; LDL, low-density lipoprotein; HDL, high-density lipoprotein; SSE, safflower seed extract; w, weeks.

stress markers (oxidized LDL and anti-MDA-LDL) or proinflammatory markers (sICAM-1, sVCAM-1, and MCP-1) were observed in either group. Anti-MDA-LDL in the SSE-supplemented group at 12 weeks tended to be lower than at baseline ( $P=0.078$ versus baseline, SSE group).

\section{Discussion}

The effect of SSE ingestion on arterial stiffness was investigated in subjects with high-normal BP or mild hypertension in this placebo-controlled, double-blind study. In a pilot study, four weeks of SSE supplementation was found to exert a favorable effect on baPWV in individuals with relatively high BP, or with a relatively high baPWV. ${ }^{18}$ Therefore, in this study, to determine the effect of SSE on baPWV precisely, we enrolled subjects on the basis of the two aforementioned inclusion criteria, and a prolonged treatment period of 12 weeks of SSE supplementation did not have a significant effect on baPWV. The nature of pulse wave velocity is significantly influenced by BP, and a pilot study suggested that SSE affects vascular function by reducing BP, which resulted in the reduction of baPWV. However, it is unclear whether reduction in BP was due to SSE supplementation, because the pilot study was an open-label, nonplacebo-controlled study. In addition, baPWV seems to be primarily associated with structural distensibility in large arteries, and long-term treatment would be required to improve baPWV. Treatment with drugs (eg, angiotensin II receptor blockers, antidiabetic agents, and eicosapentaenoic acid) were continued for $>$ six months in several clinical studies of baPWV. ${ }^{22-25}$ We suggest that a prolonged treatment period is required to reach definitive conclusions about the effect of SSE on baPWV.
SDPTG is one of the alternative methods for assessment of arterial stiffness, and this method can be used to estimate vascular age. ${ }^{16}$ It is noninvasive and easy for patients to undergo, and we consider that SDPTG is a good parameter of arterial stiffness. Some SDPTG quantifiers were found to be closely correlated with ascending aortic augmentation index, age, and other atherosclerotic risk factors. ${ }^{14-17}$ The SSE-supplemented group showed significant reduction in vascular age, as estimated by SDPTG-AI at four weeks, and showed a lower vascular age than that of the placebo group at 12 weeks. In addition, the effects of SSE were more prominent in a subgroup aged 40-59 years, although the baseline characteristics of each subgroup are similar to those of all subjects (Tables 5 and 6). The age group is a putative core target of metabolic syndrome, and a remarkable change with age has been reported for SDPTG. ${ }^{26}$ Because measurement of SDPTG is performed in peripheral vessels, it may be affected more by the peripheral artery characteristics than by pulse wave velocity. Therefore, SSE might ameliorate peripheral arterial properties and be able to detect the effects of short-term treatment.

The $\mathrm{a}$ and $\mathrm{b}$ waves corresponded to the early systolic component and the $\mathrm{d}$ wave corresponded to the late systolic component of the SDPTG. Therefore, the b/a ratio is considered to be a marker of the distensibility of large arteries (which is little affected by the reflection wave), and the $d / a$ ratio is closely related to late systolic pressure augmentation in the ascending aorta (and hence regarded as a marker of wave reflection intensity). Increase in the $b / a$ ratio and decrease in the $\mathrm{d} / \mathrm{a}$ ratio are associated with aging. The SSEsupplemented group showed a tendency towards a decreased b/a ratio $(P=0.0507$ compared with the placebo group at 
Table 5 Subject characteristics in a subgroup aged $40-59$ years

\begin{tabular}{|c|c|c|c|}
\hline & $\begin{array}{l}\text { Placebo group } \\
(n=36)\end{array}$ & $\begin{array}{l}\text { SSE group } \\
(n=34)\end{array}$ & $P$ \\
\hline Male & 30 & 28 & 1.0000 \\
\hline Female & 6 & 6 & \\
\hline Age (years) & $52.1 \pm 4.4$ & $51.0 \pm 5.6$ & 0.3437 \\
\hline Body height $(\mathrm{cm})$ & $168.23 \pm 7.36$ & $169.01 \pm 7.54$ & 0.6613 \\
\hline Body weight (kg) & $66.79 \pm 8.56$ & $67.84 \pm 10.61$ & 0.6492 \\
\hline Body mass index (\%) & $23.56 \pm 2.28$ & $23.69 \pm 2.98$ & 0.8323 \\
\hline baPWV (cm/sec) & $145 \mid .6 \pm 125.8$ & $1386.0 \pm 99.9$ & 0.0187 \\
\hline \multicolumn{4}{|c|}{ Brachial blood pressure $(\mathrm{mmHg})$} \\
\hline SBP & $133.1 \pm 13.2$ & $127.1 \pm 10.5$ & 0.0391 \\
\hline DBP & $84.6 \pm 7.6$ & $82.9 \pm 7.3$ & 0.3501 \\
\hline $\mathrm{b} / \mathrm{a}$ & $-0.543 \pm 0.112$ & $-0.556 \pm 0.143$ & 0.6527 \\
\hline$d / a$ & $-0.368 \pm 0.087$ & $-0.347 \pm 0.101$ & 0.3378 \\
\hline SDPTG-AI & $-0.25 \mathrm{I} \pm 0.263$ & $-0.292 \pm 0.302$ & 0.5470 \\
\hline Vascular age (y) & $54.5 \pm 11.0$ & $53.1 \pm 12.7$ & 0.6348 \\
\hline Alx (\%) & $84.4 \pm 13.5$ & $82.5 \pm 11.2$ & 0.5306 \\
\hline
\end{tabular}

Notes: Results are mean \pm standard deviation. Probability value refers to the difference between the treatment groups.

Abbreviations: baPWV, brachial-ankle pulse wave velocity; SBP, systolic blood pressure; DBP, diastolic blood pressure; SDPTG-AI, second derivative of a finger photoplethysmogram aging index; Alx, augmentation index; SSE, safflower seed extract.

four weeks). This may indicate improvement in distensibility of the large arteries by SSE supplementation. Katsuda et al showed that the elasticity of large arteries improved in KHC rabbits after four weeks of SSE treatment, ${ }^{9}$ and the results of their study are consistent with those of our study with regard to the effect of SSE. However, the lack of effect of SSE on baPWV, mainly reflected in the large arteries, is considered to contradict the results of $\mathrm{b} / \mathrm{a}$, as described above. This discrepancy needs to be resolved in the future.

Although there was no difference between the placebo and SSE-supplemented groups with respect to augmentation index, there was a tendency towards a reduction in augmentation index in the SSE-supplemented group at 12 weeks ( $P=0.072$ compared with baseline). The augmentation index was obtained from the central arterial waveform as the ratio of augmentation pressure to total pulse pressure. The augmentation index can also be obtained from the radial arterial waveform, and the radial augmentation index can provide information on vascular aging and arterial stiffness. Our findings of improvements in vascular age as estimated by SDPTG-AI and the augmentation index could suggest that supplementation with SSE decreases vascular aging and the risk of cardiovascular disease.

In a pilot study, Koyama et al used a daily serotonin derivative dose of $290 \mathrm{mg} /$ day. ${ }^{18}$ However, in our study we used a daily serotonin derivative dose of $70 \mathrm{mg} /$ day. The dose discrepancy between the studies could be due to the presence or absence of serotonin derivative glycosides as active compounds in SSE. We consider the effect of serotonin derivatives on arterial stiffness to be substantially dependent on aglycon type, because the bioavailability of serotonin derivative glycosides was found to be lesser than that of aglycons in rats. ${ }^{27}$ Therefore, we only analyzed the intake of serotonin derivative aglycons in this study.

Reactive oxygen species in the vessel wall have been considered to be one of the most important causes of vascular dysfunction. The oxidative modification of LDL has been shown to play a major role in the genesis of atherosclerosis. Antioxidative dietary components, such as polyphenols, have recently attracted considerable attention because they were found to be effective for the prevention of cardiovascular disease. ${ }^{28,29}$ SSE contains some serotonin derivatives, mainly CS and FS, which have been reported to act as potent free radical scavengers. ${ }^{4,5}$ Koyama et al showed that both intact and conjugated forms of CS and FS were detectable in the plasma of apolipoprotein E-deficient mice fed a diet containing SSE. Long-term supplementation of serotonin derivatives inhibited $\mathrm{CuSO}_{4}$-induced plasma lipid oxidation ex vivo and decreased the plasma titer of autoantibodies to oxidized LDL and plasma lipid peroxides in apolipoprotein E-deficient mice. ${ }^{5}$ Significant reductions in the levels of circulating oxidized LDL, anti-MDA-LDL, and urinary 8-isoprostane were observed after a four-week administration of SSE in a human pilot trial. ${ }^{18}$ In the present study, the anti-MDA-LDL titer in the SSE group was found to be lower than that in the

Table 6 Effects of SSE supplementation on vascular age in a subgroup aged 40-59 years

\begin{tabular}{|c|c|c|c|c|c|c|}
\hline & & \multicolumn{2}{|c|}{ Measurement value } & \multicolumn{3}{|c|}{ Least-squares mean change from baseline $(95 \% \mathrm{Cl})$} \\
\hline & & $\begin{array}{l}\text { Placebo group } \\
(n=36)\end{array}$ & $\begin{array}{l}\text { SSE group } \\
(n=34)\end{array}$ & Placebo group & SSE group & $\mathbf{P}$ \\
\hline \multirow[t]{4}{*}{ Vascular age (y) } & baseline & $54.45 \pm 11.02$ & $53.10 \pm 12.73$ & & & \\
\hline & $4 w$ & $57.72 \pm 10.23$ & $51.96 \pm 13.60$ & $3.39(0.74$ to 6.04$)$ & $-1.53(-4.30$ to 1.23$)$ & 0.0126 \\
\hline & $8 w$ & $57.65 \pm 10.39$ & $52.11 \pm 12.15$ & $3.25(0.78$ to 5.73$)$ & $-1.97(-4.60$ to 0.65$)$ & 0.0052 \\
\hline & $12 w$ & $56.56 \pm 11.33$ & $52.21 \pm 12.43$ & $2.1 \mathrm{I}(-0.39$ to $4.6 \mathrm{I})$ & $-2.14(-4.84$ to 0.55$)$ & $0.024 I$ \\
\hline
\end{tabular}

Notes: Results are mean \pm standard deviation. Probability value refers to the difference between the treatment groups.

Abbreviations: SSE, safflower seed extract; w, weeks; Cl, confidence interval. 
placebo group, although reduction of oxidized LDL by SSE was not detected. These data suggest that SSE could inhibit oxidized LDL formation and oxidized LDL-mediated vascular injury, and that this effect contributes to amelioration of arterial stiffness. Reduction in the level of LDL cholesterol at 12 weeks was also observed in the SSE group. However, the hypocholesterolemic effect of SSE or serotonin derivatives have not been confirmed reproducibly in animal and human studies. ${ }^{5,18}$ In addition, there is a possibility that other lipid-lowering diets influenced the results. However, we could not evaluate this possibility because we were not able to determine whether or not the subjects took a particular diet that lowers lipids. The association of hypocholesterolemic activity with the effect of SSE on arterial stiffness needs to be carefully assessed in the future.

Takimoto et al reported that CS and FS show direct vascular relaxation effects in the thoracic aorta and femoral artery of rats. ${ }^{29,30}$ Piga et al reported that CS and FS exert an inhibitory effect on short-term high glucose-induced upregulation of mRNA and proteins of adhesion and migration factors, and the consequent adhesion and migration of monocytes to endothelial cells. ${ }^{31}$ These effects may also contribute to amelioration of arterial stiffness by SSE supplementation.

This study had some limitations. First, we could not analyze smoking status in our subjects, which is an important avoidable risk factor for arterial stiffness, although subjects were advised not to smoke on the day of pulse wave analysis. Second, baPWV was significantly higher and systolic BP was marginally higher at baseline in the placebo group in this study. We do not know the exact reason for this. Third, we enrolled males aged 35-65 years and postmenopausal females aged 55-56 years in this study, and age and gender are the first variables that limit the application of such techniques. ${ }^{32}$ The consideration of the effects of SSE is limited to the group described above.

Although arterial stiffness parameters have been postulated to predict cardiovascular events, there are few interventional studies on the effect of functional food ingredients. We believe that this study may provide information regarding the clinical effects of functional food ingredients on these parameters. SSE supplementation for 12 weeks did not have any severe adverse health effects. In addition, we showed that high-dose SSE supplementation ( $210 \mathrm{mg} /$ day as serotonin derivative aglycons) for four weeks did not have severe adverse effects. ${ }^{33}$ These findings suggest that SSE may be safe for daily use as a dietary supplement over a long time period. In conclusion, this study suggests that SSE ameliorates arterial stiffness and therefore may have the potential to decrease cardiovascular risk.

\section{Acknowledgments}

The authors thank Nobuyuki Kikuchi, Osamu Mori, Takashi Kayahara, Yukihiko Shinoda, and Eiji Sukegawa for their technical assistance in this study.

\section{Disclosure}

The authors report no conflicts of interest in this work.

\section{References}

1. Bae SJ, Shim SM, Park YJ, Lee JY, Chang EJ, Choi SW. Cytotoxicity of phenolic compounds isolated from seeds of safflower (Carthamus tinctorius L.) on cancer cell lines. Food Sci Biotechnol. 2002;11(2): $140-146$.

2. Inamdar $\mathrm{V}$, Chimmad BV, Naik R. Nutrient composition of traditional festival foods of north Karnataka. J Hum Ecol. 2005;18(1):43-48.

3. Zhang HL, Nagatsu A, Watanabe T, Sakakibara J, Okuyama H. Antioxidative compounds isolated from seeds of safflower (Carthamus tinctorius L.) oil cake. Chem Pharm Bull. 1997;45(12):1910-1914.

4. Cho SH, Lee HR, Kim TH, Choi SW, Lee WJ, Choi Y. Effects of defatted safflower seed extract and phenolic compounds in diet on plasma and liver lipid ovariectomized rats fed high-cholesterol diets. J Nutr Sci Vitaminol. 2004;50(1):32-37.

5. Koyama N, Kuribayashi K, Seki T, et al. Serotonin derivatives, major safflower (Carthamus tinctorius L.) seed antioxidants, inhibit lowdensity lipoprotein (LDL) oxidation and atherosclerosis in apolipoprotein E-deficient mice. J Agric Food Chem. 2006;54(14):4970-4976.

6. Nagatsu A, Zhang HL, Mizukami H, et al. Tyrosinase inhibitory and anti-tumor promoting activities of compounds isolated from safflower (Carthamus tinctorius L.) and cotton (Gossypium hirsutum L.) oil cakes. Nat Prod Lett. 2000;14(3):153-158.

7. Takii T, Hayashi M, Hiroma H, et al. Serotonin derivatives, N- $(p$ coumaroyl) serotonin, isolated from safflower (Carthamus tinctorius L.) oil cake augments the proliferation of normal human and mouse fibroblasts in synergy with basic fibroblast growth factor (bFGF) or epidermal growth factor (EGF). J Biochem. 1999;125(5):910-915.

8. Hotta Y, Nagatsu A, Liu W, et al. Protective effects of antioxidative serotonin derivatives isolated from safflower against postischemic myocardial dysfunction. Mol Cell Biochem. 2002;238(1-2):151-162.

9. Katsuda S, Suzuki K, Koyama N, et al. Safflower seed polyphenols (N-( $p$-coumaroyl) serotonin and $\mathrm{N}$-feruloylserotonin) ameliorate atherosclerosis and distensibility of the aortic wall in Kurosawa and Kusanagi-hypercholesterolemic (KHC) rabbits. Hypertens Res. 2009; 32(11):944-949.

10. Avolio AP, Chen SG, Wang RP, Zhang CL, Li MF, O'Rourke MF. Effects of aging on changing arterial compliance and left ventricular load in a northern Chinese urban community. Circulation. 1983;68(1): 50-58.

11. Mattace-Raso FU, van der Cammen TJ, Hofman A, et al. Arterial stiffness and risk of coronary heart disease and stroke: The Rotterdam Study. Circulation. 2006;113(5):657-663.

12. Weber T, Auer J, O'Rourke MF, et al. Arterial stiffness, wave reflections, and the risk of coronary artery disease. Circulation. 2004;109(2): 184-189.

13. Chirinos JA, Zambrano JP, Chakko S, et al. Aortic pressure augmentation predicts adverse cardiovascular events in patients with established coronary artery disease. Hypertension. 2005;45(5):980-985.

14. Takada H, Washino K, Harrell JS, Iwata H. Acceleration plethysmography to evaluate aging effect in cardiovascular system. Med Prog Technol. 1997;21(4):205-210.

15. Otsuka T, Kawada T, Katsumata M, Ibuki C. Utility of second derivative of the finger photoplethysmogram for the estimation of the risk of coronary heart disease in the general population. Circ J. 2006;70(3): 304-310. 
16. Takazawa K, Tanaka N, Fujita M, et al. Assessment of vasoactive agents and vascular aging by the second derivative of photoplethysmogram waveform. Hypertension. 1998;32(2):365-370.

17. Otuka T, Kawada T, Katsumata M, Ibuki C, Kusama Y. Independent determinants of second derivative of the finger photoplethysmogram among various cardiovascular risk factors in middle-aged men. Hypertens Res. 2007;30(12):1211-1218.

18. Koyama N, Suzuki K, Furukawa Y, et al. Effects of safflower seed extract supplementation on oxidation and cardiovascular risk markers in healthy human volunteers. Br J Nutr. 2008;101(4):568-575.

19. Yamashina A, Tomiyama $H$, Takeda $K$, et al. Validity, reproducibility and clinical significance of noninvasive brachial-ankle pulse wave velocity measurement. Hypertens Res. 2002;25(3):359-364.

20. Funada J, Takata Y, Hashida H, et al. Dysfunctional central hemodynamic regulation after daily meal intake in metabolic syndrome. Atherosclerosis. 2010;210(1):268-273.

21. Bortolotto LA, Blacher J, Kondo T, Takazawa K, Safar ME. Assessment of vascular aging and atherosclerosis in hypertensive subjects: Second derivative of photoplethysmogram versus pulse wave velocity. Am J Hypertens. 2000;13(2):165-171.

22. Nakamura T, Fujii S, Hoshino J, et al. Selective angiotensin receptor antagonism with valsartan decreases arterial stiffness independently of blood pressure lowering in hypertensive patients. Hypertens Res. 2005; 28(12):937-943.

23. Sasamura H, Kitamura Y, Nakamura M, Ryuzaki M, Saruta T. Effects of the angiotensin receptor blocker candesartan on arterial stiffness and markers of extracellular matrix metabolism in patients with essential hypertension. Clin Exp Hypertens. 2006;28(5):511-520.

24. Kim SG, Ryu OH, Kim HY, et al. Effect of rosiglitazone on plasma adiponectin levels and arterial stiffness in subjects with prediabetes or non-diabetic metabolic syndrome. Eur J Endocrinol. 2006;154(3): $433-440$.
25. Mita T, Watada H, Ogihara T, et al. Eicosapentanoic acid reduces the progression of carotid intima-media thickness in patients with Type 2 diabetes. Atherosclerosis. 2007;191(1):162-167.

26. Takada H, Washino K, Harrell JS, Iwata H. Acceleration plethysmography to evaluate aging effect in cardiovascular system. Med Prog Technol. 1996-1997;21(4):205-210.

27. Shirai Y, Ueno S, Ikeuchi K, Koizumi T, Mihara R. Pharmacokinetic study of $\mathrm{N}$-feruloylserotonin and $\mathrm{N}-(p$-coumaroyl $)$ serotonin $)$ in rats. Presented at: Third International Conference on Polyphenols and Health. Tokyo, Japan, 2007 Nov 25-28.

28. Kris-Etherton PM, Keen CL. Evidence that the antioxidant flavonoid in tea and cocoa are beneficial for cardiovascular health. Curr Opin Lipidol. 2002;13(1):41-49.

29. Manach C, Mazur A, Scalbert A. Polyphenols and prevention of cardiovascular diseases. Curr Opin Lipidol. 2005;16(1):77-84.

30. Takimoto T, Arisaka H, Suzuki K, Murata T, Ozaki H, Koyama N. Antiatherosclerotic action of safflower seed polyphenols (N- $(p-$ coumaroyl) serotonin), N-feruloylserotonin) on vascular smooth muscle cell [abstract]. J Clin Biochem Nutr. 2007;41 (Suppl):117.

31. Piga R, Naito Y, Kokura S, Yoshikawa T. Inhibitory effect of serotonin derivatives on high glucose-induced adhesion and migration of monocytes on human aortic endothelial cells. Br J Nutr. 2009;102(2): 264-272.

32. Wang X, Xie J, Zhang LJ, Hu DY, Luo YL, Wang JW. Reference values of brachial-ankle pulse wave velocity for Northern Chinese. Chin Med J. 2009;122(18):2103-2106.

33. Suzuki K, Koyama N, Sukegawa E, et al. Evaluation of safety of excessive intake of safflower seed extract dietary supplement in healthy adult subjects. Seikatsu Eisei. 2009;53(2):100-109.
Vascular Health and Risk Management

\section{Publish your work in this journal}

Vascular Health and Risk Management is an international, peerreviewed journal of therapeutics and risk management, focusing on concise rapid reporting of clinical studies on the processes involved in the maintenance of vascular health; the monitoring, prevention and treatment of vascular disease and its sequelae; and the involvement of

\section{Dovepress}

metabolic disorders, particularly diabetes. This journal is indexed on PubMed Central and MedLine. The manuscript management system is completely online and includes a very quick and fair peer-review system, which is all easy to use. Visit http://www.dovepress.com/ testimonials.php to read real quotes from published authors. 